\title{
Middle Ear Effusion, Attention, and the Development of Child Behavior Problems*
}

\author{
Jannette Cross ${ }^{1}$, Dale L. Johnson ${ }^{1}$, Paul Swank ${ }^{2}$, Constance D. Baldwin ${ }^{3}$, David McCormick ${ }^{4}$ \\ ${ }^{1}$ University of Houston, Houston, USA; ${ }^{2}$ University of Texas Medical School, Houston, USA; ${ }^{3}$ University of Rochester, Rochester, \\ USA; ${ }^{4}$ University of Texas Medical Branch, Galveston, USA. \\ Email: dljohnson27@msn.com
}

Received April $7^{\text {th }}, 2010$; revised June 23 ${ }^{\text {rd }}, 2010$; accepted June $25^{\text {th }}, 2010$.

\begin{abstract}
Objective: Much interest centers on whether middle ear effusion (MEE) early in life has lasting developmental consequences. It was hypothesized that episodic loss of hearing acuity associated with MEE results in a deficit in attention, a core factor in the development of child behavior problems, and that impaired attention is related to behavior problems during the early years of childhood. Method: This was a prospective study of a large sample of children $(n=698)$ that was representative of the local population in terms of socioeconomic and ethnic characteristics. The children were recruited at birth and were monitored with regular home visits for 3 years to check for the presence of MEE. Assessment of attention occurred at 2, 3, 5, and 7 years. Behavior problems were assessed at 3, 5, and 7 years. Results: The results did not support the hypothesis that children with greater duration of MEE experience greater attention deficits and more behavior problems than children with a shorter duration of MEE. Structural Equation Modeling parameter estimates resulted in no support for the primary hypothesis. Correlational analyses also did not support the hypothesis. Attention and behavior problems were related significantly. Conclusions: Our negative findings call into question the results of previous studies relating MEE to behavior and attention problems, studies that may have been biased by small, non-representative samples and retrospective designs that lacked careful documentation of MEE.
\end{abstract}

Keywords: Middle Ear Effusion, Attention, Behavior Problems

\section{Introduction}

Middle ear effusion (MEE), an inflammation of the middle ear accompanied by effusion or a collection of liquid in the middle ear, is one of the most commonly diagnosed illnesses among young children. Estimates of MEE incidence rates in the United States range from $49 \%$ to $97 \%$ during the first year of life [1]. Incidence peaks between 6 and 18 months with a steady decline until around 5 years, when there is a second smaller peak $[1,2]$. Almost one-third of all children suffer with chronic MEE and it is estimated that some spend an average of $38 \%$ to $70 \%$ of their first 3 years with MEE [3]. Most children with MEE have an average hearing loss of 20 to $30 \mathrm{~dB}$ [4] during an episode. The conductive hearing loss associated with MEE causes sounds to be muffled and distorted.

The fluctuating hearing loss caused by episodes of

*This project was supported under award \# HD20988 R01, from the National Institute of Child Health and Development, "Impact on Child Development of Early Otitis Media".
MEE is believed to cause developmental problems, including child behavior problems. Problems in language and speech have been the focus of much research $[5,6]$ and it has also been proposed that attention processes may be effected [7]. It has been hypothesized that frequent episodes of MEE during early childhood produce initial language delays and a reduction in attention-to-language. In the later preschool years, when the number of MEE episodes is reduced and hearing returns to normal, basic language skills recover. However, children with recurrent MEE may not be able to attend to language input consistently and may develop the habit of not attending because of the greater effort required. This, in turn, may lead to an attention deficit for language-related tasks that require sustained attention, a deficit that persists after hearing is normal $[8,9]$. In addition to effects on language and attention, behavior problems may ensue. Children may withdraw and become less responsive to their environment, or act out because they cannot respond to the positive cues in the environ- 
ment. Parents and teachers may have more difficulty providing responsive stimulation. They may perceive the child as willfully ignoring them, and change their own interactive behavior accordingly [10].

\subsection{MEE and Attention}

Several prospective studies have found persistent MEE and attention to be linked [7-11]. In a prospective study with a sample size of 433, Mohr-Sperduti [12] found that children with recurrent MEE showed an enduring shift in temperament. MEE was negatively associated with two dimensions of temperament: attention and difficult/fussiness.

Four studies did not find an association of MEE to attention or obtained mixed results [13-16]. It should be noted that sample sizes were small in these projects and they used different measures of attention from those that had positive results.

There is evidence that MEE and attention are related, particularly during the early years or during periods of active MEE. However, because of methodological issues, verification of a causal relation between MEE and attention remains inconclusive. Most of the retrospective and all of the prospective studies except the Mohr-Sperduti [12] study used small samples. Many of the samples involved special populations that were defined by socioeconomic status or clinical status. These studies used a variety of measures of attention, from rating scales to observational techniques and continuous performance tasks.

\subsection{MEE and Behavior Problems}

Four research groups have carried out prospective studies of MEE and behavior problems. Silva et al. [11] and Bennett et al. [17] found an association between MEE and behavior problems; but Roberts et al. [16] and Paradise et al. [18] did not. MEE assessment for the Bennett and Silva projects was conducted at 5 years of age and the Roberts and Paradise projects examined ears in the first 3 years. Silva assessed behavior problems at age 5 years and Bennett at age 10 years. Roberts assessed behavior problems at age 12 years and Paradise at age 4 years.

Although evidence is limited, results of some studies support the hypothesis that MEE is associated with the development of behavior problems. In addition to being few in number, these studies include populations of limited generalizability and small samples. The question of how MEE is related to behavior problems remains unresolved.

\subsection{Attention}

Although there is considerable agreement that attention plays a role in the development of behavior problems in children, research on the issue has been slowed by a lack of consensus about the definition and measurement of attention [19]. Children with attention problems are noted to be inconsistent in their behavior over time or across situations in the performance of socialization, communicative, and self-care skills at age appropriate levels, despite generally average intelligence levels [20, 21]. One problem for researchers is that attention measures are only moderately stable over time $[22,23]$. Measures of inattention tend to be more stable than those of attention [23,24]. That attention is a complex and multifactorial process has led to the development of a wide range of measures. Mirsky [25] suggested that the multifaceted nature of attention and the different approaches often used to assess attention from study to study could account for the variability in outcome among studies of attention.

\subsection{Attention and Behavior Problems}

Research with children who have been referred for treatment of behavior problems, attention deficit disorder or learning disorders has found a consistent association between attention difficulties and behavior problems [2631]. These studies tend to examine the relation between attention and behavior problems using concurrent assessment of the variables and to include samples of special populations and school-age children. We found no prospective studies of young children outside the clinical setting. We also found no studies of MEE, attention and behavior problems.

\subsection{Research Hypotheses}

Based on our review of the literature we formed two hypotheses: 1) attention difficulties are core factors in the development of child behavior problems, and attention is affected adversely by the hearing loss associated with persistent or intermittent MEE during the early years of childhood; 2) the relation of MEE and, therefore, attention, on behavior problems would exhibit a stronger effect at 3 years of age, a time when the child would more recently have experienced moreMEE, than at 7 years. Improvement is expected because of the return of normal hearing as episodes become less frequent during the later preschool years. With advancing age, children have time for adaptation and previous deficits become less severe or disappear altogether.

Also this study examined whether duration of MEE was related directly to child behavior problems at ages 3 and 7 and to attention problems at these ages. Finally, we asked whether SES, home environment, or gender moderate the negative effect of MEE. For example, children with persistent MEE and low attention, but who are reared in positive and highly stimulating environments, may not develop more behavior problems due to the buffering effects of positive environmental stimulation. 


\section{Method}

\subsection{Sample}

The study was part of a longitudinal study of middle ear effusion and its effects on child development. Infants ( $\underline{n}$ $=698$ ) were recruited at birth from two hospital newborn nurseries in the Galveston and the near mainland Texas area. Only normal term infants were included. Low birthweight babies, babies with known neurological disorders and babies from families whose primary language was not English were excluded. Subjects were recruited while the mother and baby were still in one of the maternity units. Written informed consent from parents was obtained. Parents agreed to have a research assistant come to their homes on a frequent basis to check the child's ears and agreed to come into the research center for more psychological assessments during the first few years of their child's life. Parents were paid for their participation at each of the major assessments conducted at the research center at 2,3,5, and 7 years. The payment was $\$ 35$ early in the project was later raised to $\$ 50$. In almost all cases, these assessments occurred within 4 weeks of the child's birthday.

This project was approved by institutional review boards at both the University of Texas Medical Branch and the University of Houston.

\subsection{Procedures}

MEE status was assessed regularly from birth to 3 years. At 2, 3, 5, and 7 years, a battery of cognitive, language and behavioral assessments was administered to children at the research center at the University of Texas Medical Branch in Galveston. Examiners were four graduate students in psychology (3) and in speech communication disorders (1) who were especially trained in administration of the tests. Only children with normal hearing as indicated by audiometric testing carried out at the time of the examinations were tested. All children but one had normal hearing and this one was dropped from the study. Examiners were blind to the MEE condition of the children.

The participant's gender and ethnicity were obtained by parental report at the time of enrollment at birth. At 2 years, data on socioeconomic status (SES) and level of educational stimulation in the home environment were collected. Assessment of attention was obtained through parent report questionnaires at 2, 3, and 5 years. Examiner rating of attention occurred at 3,5, and 7 years. At 7 years, a computerized continuous performance task assessed attention. Behavior problems were assessed with parent report questionnaires at 3,5 and 7 years, and with teacher reports at 5 and 7 years.

\subsection{Measures}

Variables included in this study of children were MEE, infant and young child attention, and young child emotional and behavioral problems. Control variables included gender, SES, and Home Observation of the Measured Environment (see below).

\subsection{MEE}

MEE status was defined as the presence of otitis media with effusion independent of other symptoms. Every 2 to 4 weeks from birth to 18 months and every 4 weeks from 18 to 36 months, scheduled visits at the home or daycare were conducted by trained technicians to monitor presence of MEE with tympanometry [3]. Acoustic reflectivity was measured at the initial $30 \%$ of the visits, but was replaced with tympanometry, which was performed at all visits. Diagnosis of MEE occurred if either ear met any of these criteria: (1) presence of otorrhea, or purulent pus draining from the ear canal visible without otoscopy; (2) acoustic reflectivity $\geq 5$; or (3) a Type B tympanogram (i.e., compliance of 0.0 or 0.1 , or compliance of 0.2 or 0.3 only if the absolute gradient was $<0.1 \mathrm{ml}$ ). For children with tympanostomy tubes, a diagnosis of MEE was made by the presence of purulent otorrhea or a Type B tympanogram in the presence of an external ear canal volume which indicates that the tube was not patent. At each home visit, both ears were evaluated by this criteria and categorized at either "normal" or "MEE". A computer-generated algorithm calculated the percentage of time each child spent with MEE for a given period (total days with MEE/total days in the examination period). To calculate time with MEE, two consecutive visits positive for MEE would equal 28 days with MEE. If only one visit of the two consecutive visits was positive for MEE, half of the intervening days (up to 14) were counted as days with MEE. This resulted in a maximum of 28 days of MEE was counted for any one MEE-positive visit [32]. Unilateral, bilateral or combined days of MEE were calculated. Parents were informed of the child's middle ear status and encouraged to see a physician if MEE was diagnosed. Duration of time and proportion of time spent with bilateral MEE was calculated for each child at 6,12 , 18,24 , and 36 months. The two measures were highly correlated and proportion of time was used for the analyses.

\subsection{Attention}

The strategy we adopted for the measurement of attention was to use four different measures that were ageappropriate; 1) parent ratings, 2) teacher rating, 3) examiner observation, and 4) computerized test.

Attention was assessed with parent report at 2, 3, and 5 years with the Task Orientation scale of the Revised Dimensions of Temperament Survey (DOTS-R) [33]. The alpha coefficient for preschool children was .79. At 3, 5, and 7 years, upon completing the cognitive assessment with the Stanford-Binet Intelligence Scale: Fourth Edi- 
tion [34], examiners rated children's attention with two items on the Stanford-Binet for examiners' ratings of overall test performance: a) absorbed by task; and b) persistent. Examiners were trained by having pairs of examiners rate the same test-taking behavior. This was continued until ratings agreed $80 \%$ of the time. There was a follow-up repetition of this procedure to check on persistence of agreement. At 5 and 7 years, attention was assessed with parent report using the Attention scale of the Child Behavior Checklist for Ages 4-16 [35]. The Attention scale of the Teacher Report Form [36] was used to assess teacher-report of attention at 5 and 7 years.

At 7 years, attention was assessed by a computerized continuous performance test, the Test of Variables of Attention (TOVA) [37]. The 5-year-old test length of 11 minutes was used to save time in a lengthy battery of tests and because it was found to be highly correlated with the longer version. In addition, children found the longer version unbearably tedious. The test was always administered last. Four TOVA variables were used: 1) Errors of Omission or failure to respond to a target; 2) Errors of Commission or responding to the non-target; 3) Mean Correct Response Times; and 4) Variability, the standard deviations of response times. The occurrence of errors of omission was considered an indication of inattention whereas the occurrence of errors of commission was an indicator of impulsivity. Mean correct response time was an indicator of processing and response time. Variability relates to consistency in speed of responding.

\subsection{Behavior Problems}

Behavior problems were assessed by parent report at 3 years with the Child Behavior Checklist for Ages 2-3 (CBCL/2-3) [35] and at 5 and 7 years with the Child Behavior Checklist for Ages 4-16 (CBCL/4-16) [36]. At 5 and 7 years, teachers completed the Teacher's Report Form (TRF) [36]. Attention scale items are not included in either the Internalizing or Externalizing factors of the CBCL that are used as dependent variables. The Internal scale includes depression and anxiety and the External scale includes acting out disorders. One-week test-retest reliability for the Total Problems for the CBCL/2-3 was $r$ $=0.91$ and for the $\mathrm{CBCL} / 4-16$ it was $\mathrm{r}=0.93$. The CBCL and TRF were used because they were measures most often used by behavior problem researchers and the measures are well-standardized and have good reliability and validity $[35,36,38]$.

\subsection{Moderating Variables}

The Hollingshead Four-Factor Index of Social Status was used to assess family SES [39]. This index makes use of parental occupation and education. Educationally stimulating characteristics of the home environment were assessed with the Home Observation for Measurement of Environment Inventory (HOME) [40]. The mother was interviewed and observed with her child in the home. The Total Score was used.

\subsection{Reliabilities}

All measures used had adequate reliability. Details may be found in the key manuals for each measure.

\subsection{Statistical Analysis}

Descriptive analyses of each variable were conducted. Bivariate correlations among all variables were computed. To test the overall hypothesized relation between MEE, attention and behavior problems, structural equation modeling (SEM) using LISREL 7 [41,42] was used. SEM was selected because of its ability to run multiple paths simultaneously and to test for possible networks of causality, using goodness of fit test for evaluation. Also, because SEM examines relations among latent variables, the errors in the indicators are eliminated giving more powerful tests of the hypotheses. See Figure 1 for the hypothesized model. Only attention and behavior variables at 3 and 7 years were included, omitting data available at 5 years in the SEM. This was done to reduce the number of path estimates to compensate for the sample size, which was considered marginal for SEM. Goodness of fit indices and parameter estimates between the hypothesized model and comparison models were examined.

\section{Results}

From the initial sample of 698 recruited at birth, attrition occurred because families moved out of the research area or lost interest in participation and for various other reasons. One child was excluded after documentation of a sensory-neural hearing loss. The demographics for the sample initially recruited and at age 7 are as follows: female, $51 \%$ and $50 \%$; African-American, $32 \%$ and $31 \%$, Euro-American, $55 \%$ and $53 \%$, and Hispanic, $14 \%$ and $16 \%$; mothers married or co-habiting, $72 \%$ and $69 \%$; Hollingshead Socioeconomic Status mean 37 (s. d., 13) and $37 \%$ (s. d. 13), and HOME mean, 39 (s. d. 5) and 39 (s. d. 5). Demographic characteristics of the sample remaining after attrition were very similar. Further evidence of the normal distribution of scores for this sample, and the normality of the sample, may be seen in the results for the Stanford-Binet Fourth Edition [34] administered to children at ages 3,5 and 7. Their IQ scores were, 100.6 (SD = 12.2), 98.3 (12.6), and $98.0(14.00)$, respectively, which are close to the normative sample IQ of 100.0 $(\mathrm{SD}=15.0)$. Sample sizes at the evaluation time points were approximately 395 at age 2, 360 at age 3, 310 at age 5 and 200 at age 7.

We found no significant correlations between any of the MEE by time period and behavior problem scales. Correlations ranged from -0.14 to 0.11 . Correlations between MEE and attention ratings were also low. Of 24 


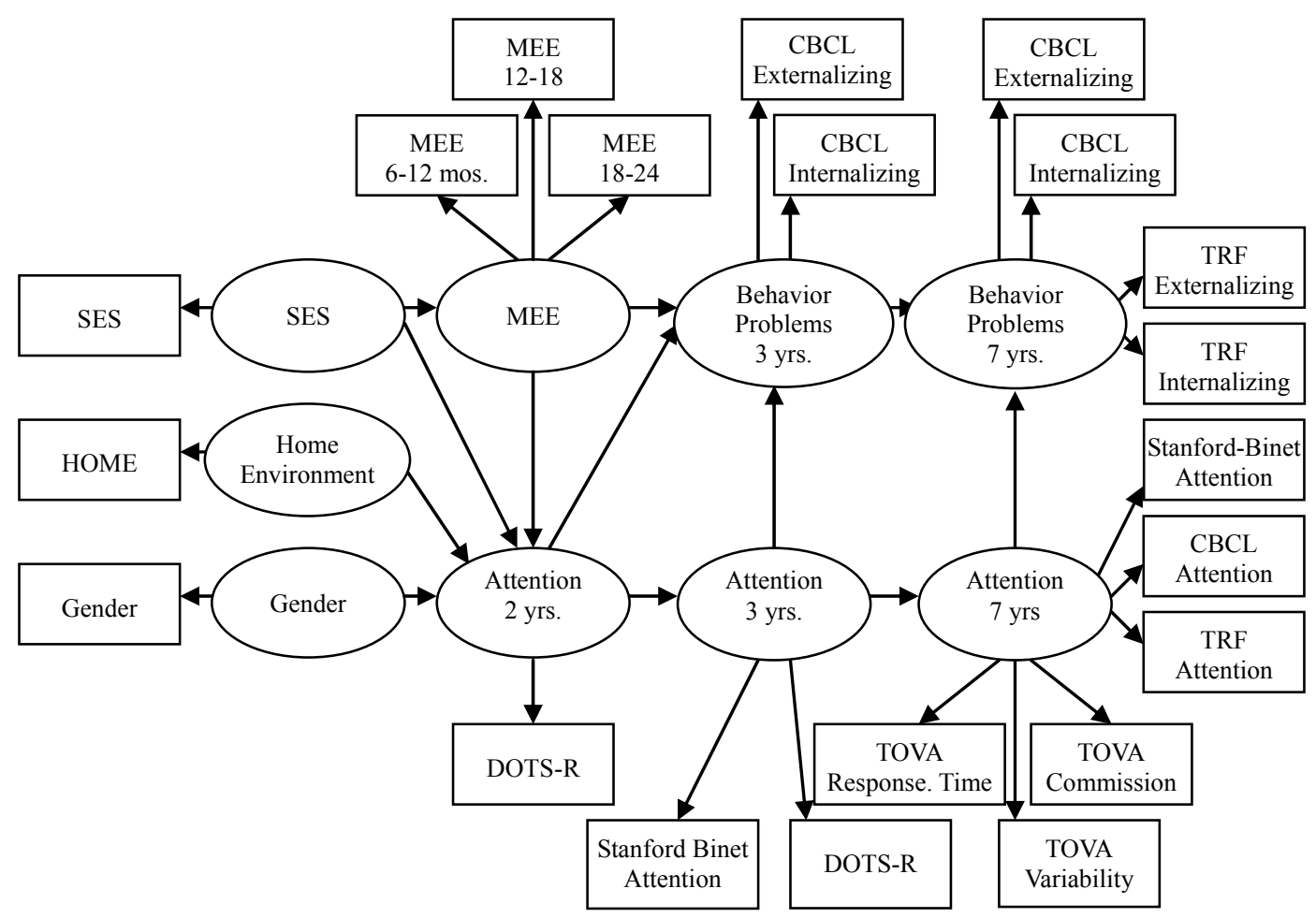

Figure 1. Hypothesized structural equation modeling

correlations, only two were significant. One was for Stanford-Binet-Attention (SB-Attn) and MEE, $r=0.14, p$ $<0.05$. MEE and continuous performance task (TOVA) correlations were non-significant for all TOVA measures except Variability. The significant MEE correlation with Variability was $\mathrm{r}=-0.21, \mathrm{p}<0.01$.

Attention ratings and behavior problems were significantly related. For the CBCL Externalizing, Internalizing and Total scores at age 3, five of six correlations with DOTS were significant (range: $\mathrm{r}=-0.14$ to -0.25$)$ ). None were significant at age 7. All 6 of the CBCL attention ratings at ages 5 and 7 were significantly related to CBCL scores at age 7 (range, $r=0.38$ to 0.80 ). None of the TOVA scores were related to CBCL scores at age 7. Using the CBCL Attention scale with a cut-off of 68 at age seven $4.4 \%$ of the children were found to have attention problems. The teacher version showed $4.5 \%$ with such problems. There were no gender differences.

For the primary inferential analysis, structural equation modeling (SEM) was used to test the adequacy of the theoretical model. SEM was selected because of its ability to run multiple paths simultaneously and to suggest a possible network of causal relations. The first step was model specification. Initial interest was in obtaining an adequate measurement model between the observed variables and the latent variables. However, the hypothesized measurement model could not be identified and major revisions were made before testing the structural model of relations between the latent variables. The first step involved elimination of observed variables in order to increase covariances among variables within each construct. The SB-Attn at 3 and 7 years and the TOVA variables of Omission and Variability were eliminated. The latent variable for Attention at 7 years was divided into two separate latent variables. One consisted of CBCL and TRF Attention scales and the other consisted of the two remaining TOVA variables, Omission and Variability. In addition, SES and HOME were changed from estimating two separate latent variables to estimating one latent variable of environment. Lastly, the disturbance terms for CBCL Attention at 7 years and $\mathrm{CBCL}$ Externalizing at 7 years and for TRF Attention and TRF Externalizing were allowed to be correlated to account for method effects. These modifications did produce a workable model, but one that still did not meet measurement requirements according to the chi-square results. Additional changes were made based on examination of the fitted residuals and modification indices which lead to the removal of the observed variables, TRF and CBCL Internalizing at 7 years. The revised measurement model resulted in a large improvement in chi-square, goodness-of-fit and adjusted goodness-of-fit. The root mean square residual also decreased slightly. Any further changes would have resulted in major deviations from the hypothesized measurement model.

Once an adequate measurement model was established, the next step involved model identification and parameter estimation of the structural model based on maximum- 
likelihood estimates from the covariance-variance matrix with pairwise deletion of missing values. Significance of parameter coefficients was based on the ratio of the statistic to its standard error, which are asymptotically normal. $Z$ values $<-2$ and $>2$ are considered significant. This model had direct effects from MEE to each of the latent endogenous variables. Home environment and gender also had direct effects on attention and behavior problem variables. However, no solution convergence was obtained. Parameters from the exogenous variables were redefined to allow a direct effect on each of the endogenous variables. This change resulted in model convergence.

The hypothesized structural model with parameter estimates is presented in Figure 1. A summary of the specification and fit indices for the hypothesized model is presented in Table 1. Regarding the primary research hypothesis that children with persistent MEE tend to have attention deficits and that deficits in attention result in more behavior problems, examination of parameter coefficients did not support that MEE has an effect on either attention or behavior problems. Between attention and behavior problems, there was one significant parameter estimate from Attention-A (CBCL and TRF) and Behavior Problems at 7 years. This finding also did not support a secondary research hypothesis that proposed stronger relations between MEE, attention, and behavior problems at 3 years than at 7 years. Lastly, regarding the hypothesis that SES, home environment and gender would moderate the negative effect of MEE, several significant parameter estimates were found for Home Environment (SES and HOME) predicting behavior problems at 3 years and to deficits in Attention A (CBCL and TRF) at 7 years. In addition, significant parameter estimates were found from gender to both endogenous attention variables at 7 years. Significant relations are marked with asterisks (Figure 2).

\section{Discussion}

This study provided a prospective examination of the impact of MEE on attention and behavior problems. It was hypothesized that a deficit in attention is a core fac-

Table 1. Summary of specification and fit statistics for structural equation models of MEE, attention and behavior problems

\begin{tabular}{lcccccc}
\hline Measurement Model & $\mathrm{t}^{2}$ & $\mathrm{df}$ & $\mathrm{p}$ & GFI & AGFI & RMSEA \\
\hline Revised Model A & 235.77 & 93 & 0.00 & 0.8699 & 0.7608 & 0.0881 \\
Revised Model B & 75.10 & 69 & 0.2875 & 0.9434 & 0.8884 & 0.0157 \\
\hline Structural Model & $\mathrm{t}^{2}$ & $\mathrm{df}$ & $\mathrm{p}$ & GFI & AGFI & RMSEA \\
\hline Hypothesized & 84.4114 & 80 & 0.3464 & 0.9359 & 0.8910 & 0.0118 \\
Comparison A & 84.7454 & 82 & 0.3959 & 0.9355 & 0.8930 & 0.0041 \\
Comparison B & 85.4187 & 84 & 0.4363 & 0.9347 & 0.8943 & 0.0 \\
\hline
\end{tabular}

Note: GFI = goodness of fit index; AGFI $=$ adjusted goodness of fit index; RMSEA = root mean square error of approximation.

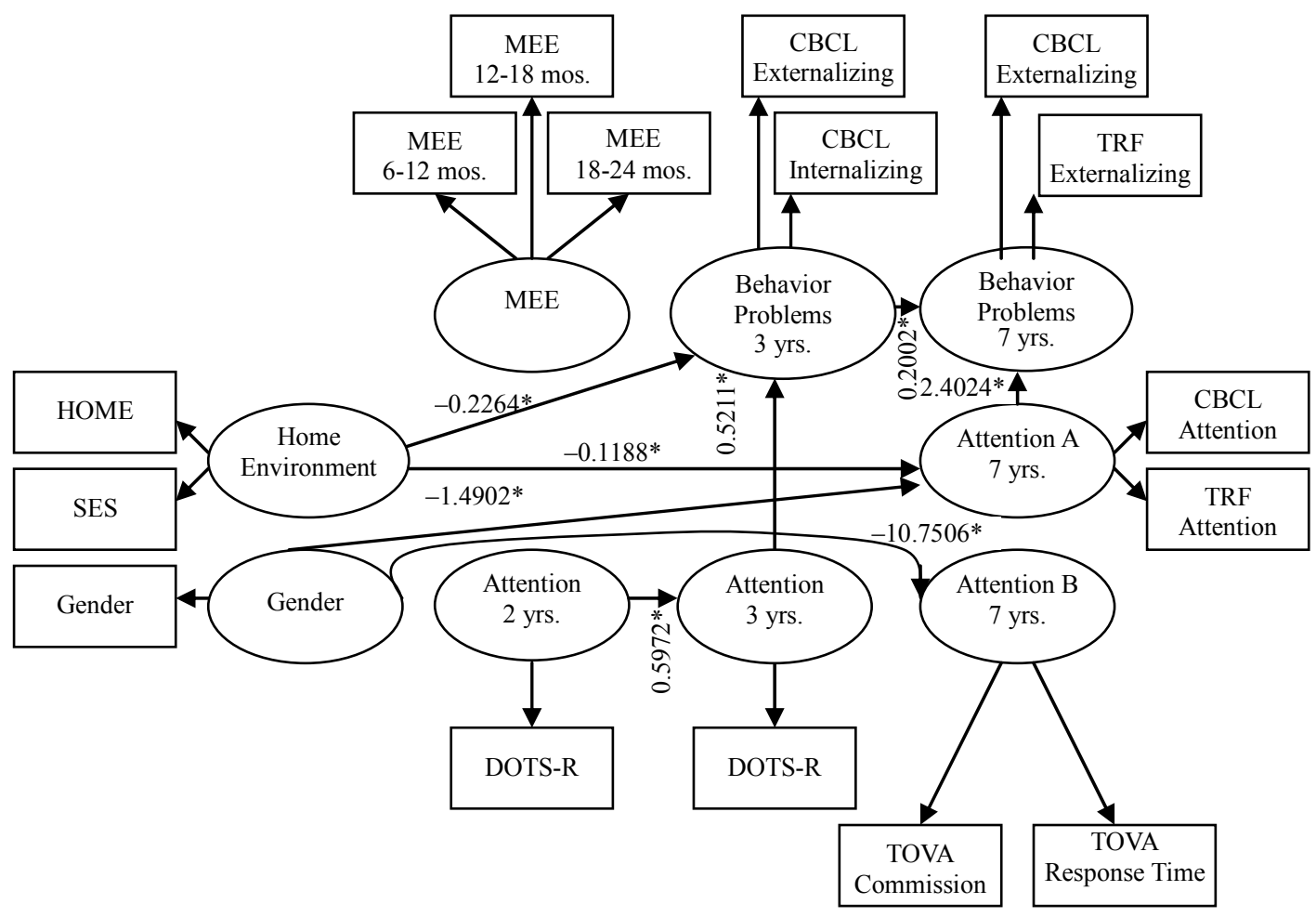

Figure 2. Significant parameters for comparison structural equation: Model B 
tor that contributes to the development of a range of behavior problems and that attention is adversely affected by persistent MEE. Important strengths of the present study are its prospective design, early and regular monitoring of MEE, and selection of a large non-clinical sample representative of the general population. These characteristics contrast with those of the majority of studies using retrospective methods to investigate the relation of MEE to either attention or behavior problems [43]. Most had small samples [7-10,13-15,43-46]. Only a few studies relied on a large sample $[11,12,18]$. Often the samples were limited to low SES subjects $[7,9,10,13,14,16]$, children with restricted SES representation [8], or to children referred for clinical evaluation $[7,44]$. Thus, this study provides an important perspective on the relations between MEE, attention, and behavior problems because of its methodological strengths.

The results of this study generally failed to support the hypothesis that children with greater duration of MEE experience greater attention deficits and more behavior problems than children with a shorter duration of MEE. SEM parameter estimates provided no support for the first hypothesis. Correlational analyses also did not support the hypothesis. Correlations between MEE and attention resulted only in association between examiner ratings of attention at 7 years and earlier MEE. Also TOVA Variability at 7 years was associated negatively with MEE, instead of positively, as expected. These two correlations were small and could be attributed to the increase of a Type I error due to familywise error. In addition, there were no significant associations between MEE and behavior problems, even at 3 years of age.

These results are consistent with those obtained by Roberts et al. [16] and Paradise et al. [18] who used prospective methods to identify duration of MEE in the first three years. Roberts et al. [18] found that duration of MEE was not related to number of behavior problems at 12 years of age. On the other hand, the present results contrast with those of Silva and associates [11], who did find persistent MEE to be related to more behavior problems. The Silva group used a cross-sectional method with behavior problems assessed at age 5. Having impaired hearing at that age was related to behavior problems and this relation was still present at ages 7, 9, and 11 [48].

The second hypothesis that attention mediates the effects of MEE on behavior problems was not supported. MEE was not related to either attention or behavior problems. However, some support was found for a relation between attention and behavior problems, excluding the role of MEE. Examination of individual parameters indicated that impaired attention at 7 years was related to behavior problems at 7 years, with both variables assessed by parent and teacher report. The second hypothe- sis, which proposed that there would be stronger effects of MEE on behavior problems at age 3 than at age 7, was not supported. There were no effects at either age.

An important limitation of this study derived from the LISREL analysis that demonstrated a lack of association among variables within constructs and between the constructs. The originally hypothesized measurement model required significant revisions in order to get a solution to converge, resulting in a weak fitting measurement model. The small to moderate correlations among the measures of attention and behavior problems call into question the construct validity of the measures. Within attention measures, although some significant correlations were found across parent, teacher and examiner reports, these correlations were low.

Given these methodological limitations, our results lead to the cautious conclusion that MEE in the first 3 years of life is not related to behavior problems that are present at ages 3 and 7 .

\section{REFERENCES}

[1] K. A. Daly, "Definition and Epidemiology of Otitis Media," In: J. E. Roberts, I. F. Wallace and F. W. Henderson, Eds., Otitis Media in Young Children: Medical, Developmental, and Educational Considerations, Brookes Publishing, Baltimore, 1997.

[2] G. A. Zielhuis, G. H. Rach, A. van den Bosch, et al., "The Prevalence of Otitis Media with Effusion: A Critical Review of the Literature," Clinical Otolaryngology, Vol. 15, No. 3, 1990, pp. 283-288.

[3] M. Owen, C. D. Baldwin, D. Luttman, et al., "The Universality of Otitis Media with Effusion Detected by Tympanometry on Frequent Home Visits in Galveston, Texas," Proceedings of the 5th International Symposium on Recent Advances in Otitis Media, Fort Lauderdale, 1993.

[4] P. D. Eimas and J. F. Kavanagh, "Otitis Media, Hearing Loss, and Child Development: A NICHD Conference Summary," Public Health Reports, Vol. 101, No. 3, 1986, pp. 289-293.

[5] D. P. McCormick, C. D. Baldwin, J. S. Klecan-Aker, et al., "Association of Early Bilateral Middle Ear Effusion with Language at Age 5 Years," Ambulatory Pediatrics, Vol. 1, No. 2, 2001, pp. 87-90.

[6] D. J. Lim, C. R. Bluestone, J. O. Klein, et al., "Recent Advances in Otitis Media," Decker Periodicals, Philadelphia, 1993.

[7] J. S. Gravel and I. F. Wallace, "Listening and Language at 4 Years of Age: Effects of Early Otitis Media," Journal of Speech and Hearing Research, Vol. 35, 1992, pp. 588595.

[8] L. V. Feagans, E. Kipp and I. Blood, "The Effects of Otitis Media on the Attention Skills of Day-Care-Attending Toddlers," Developmental Psychology, Vol. 30, No. 5, 1994, pp. 701-708.

[9] L. V. Feagans, M. Sanyal, F. Henderson, et al., "Rela- 
tionship of Middle Ear Disease in Early Childhood to Later Narrative and Attention Skills," Journal of Pediatric Psychology, Vol. 12, No. 4, 1987, pp. 581-594.

[10] J. E. Roberts, M. R. Burchinal and F. Campbell, "Otitis Media in Early Childhood and Patterns of Intellectual Development and Later Academic Performance," Journal of Pediatric Psychology, Vol. 19, No. 3, 1994, pp. 347-367.

[11] P. A. Silva, C. Kirkland, A. Simpson, et al., "Some Developmental and Behavioral Problems Associated with Bilateral Otitis Media with Effusion," Journal of Learning Disabilities, Vol. 15, No. 7, 1982, pp. 417-421.

[12] S. M. Sperduti, “A Structural Model for Otitis Media and Pain Effects on Attention and Difficult/Fussiness Factors of Temperament," Unpublished Masters Thesis, University of Houston, Houston, 1996.

[13] E. Arcia and J. E. Roberts, "Otitis Media in Early Childhood and its Association with Sustained Attention in Structured Situations," Behavioral Pediatrics, Vol. 14, No. 3, 1993, pp. 181-183.

[14] J. Kindig, "Otitis Media: Its Relationship with Delayed Reading and Attention Deficit Disorder," Dissertation Abstracts International, Vol. 56, 1996, p. 3520.

[15] K. R. Minter, J. E. Roberts, S. R. Hooper, et al., "Early Childhood Otitis Media in Relation to Children's Attention-Related Behavior in the First Six Years of Life," Pediatrics, Vol. 107, No. 5, 2001, pp. 1037-1042.

[16] J. Roberts, M. R. Burchinal and S. M. Clarke-Klein, "Otitis Media in Early Childhood and Cognitive, Academic, and Behavior Outcomes at 12 Years of Age," Journal of Pediatric Psychology, Vol. 20, No. 5, 1995, pp. 645-660.

[17] K. E. Bennett, M. P. Haggard, P. A. Silva, et al., "Behaviour and Developmental Effects of Otitis Media with Effusion into the Teens," Archives of Disease in Childhood, Vol. 85, No. 2, 2001, pp. 91-95.

[18] J. L. Paradise, C. A. Dollaghan, T. F. Campbetll, et al., "Otitis Media and Tympanostomy Tube Insertion during the First Three Years of Life: Developmental Outcomes at the Age of Four Years," Pediatrics, 2003, Vol. 112, No. 2, pp. 265-277.

[19] M. K. Rothbart, M. L. Posner and K. L. Hershey, "Temperament, Attention and Developmental Psychopathology," In: D. Cicchetti and D. J. Cohen, Ed., Developmental Psychopathology, Theory and Methods, John Wiley, New York, Vol. 1, 1995, pp. 315-340.

[20] R. A. Barkley, G. J. DuPaul and M. McMurray, "Comprehensive Evaluation of Attention Deficit Disorder with and without Hyperactivity as Defined by Research Criteria," Journal of Consulting and Clinical Psychology, Vol. 58, No. 6, 1990, pp. 775-789.

[21] NJ Roizen, TA Blondis, M Irwin, et al., "Adaptive Functioning in Children with Attention-Deficit Hyperactivity Disorder," Archives of Pediatrics \& Adolescent Medicine, Vol. 148, No. 11, 1994, pp. 1137-1142.

[22] R. J. McGowan, D. L. Johnson and S. E. Maxwell, "Relations between Infant Behavior Ratings and Concurrent and Subsequent Mental Test Scores," Developmental Psychology, Vol. 17, No. 5, 1981, pp. 542-533.
[23] H. Ruff, K. Lawson, R. Parrinello, et al., "Long-Term Stability of Individual Differences in Sustained Attention in the Early Years," Child Development, Vol. 61, No. 1, 1990, pp. 60-75.

[24] C. M. Heinicke, S. D. Diskin, D. M. Ramsey-Klee, et al., "Pre- and Post-Birth Antecedents of 2-Year-Old Attention, Capacity for Relationships and Verbal Expressiveness," Developmental Psychology, Vol. 22, No. 6, 1986, pp. 777787.

[25] A. F. Mirsky, "Behavioral and Psychophysiological Markers of Disordered Attention," Environmental Health Perspectives, Vol. 74, 1987, pp. 191-199.

[26] S. B. Campbell, "Longitudinal Studies of Active and Aggressive Preschoolers: Individual Differences in Early Behavior and in Outcome," In: D. Cicchetti and S. L. Toth, Eds., Rochester Symposium on Developmental Psychopathology: Internalizing and Externalizing Expressions of Dysfunction, Erlbaum Group, Hillside, Vol. 2, 1991.

[27] M. J. Eliason and L. C. Richman, "Behavior and Attention in LD Children," Learning Disability Quarterly, Vol. 11, 1988, pp. 360-369.

[28] G. W. Rebok, W. E. Hawkins, P. Krener, et al., "Effect of Concentration Problems on the Malleability of Children's Aggressive and Shy Behaviors," Journal of the American Academy of Child and Adolescent Psychiatry, Vol. 35, No. 2, 1996, pp. 193-203.

[29] K. S. Lassiter, R. C. D’Amato, D. J. Raggio, et al., "The Construct Specificity of the Continuous Performance Test: Does Inattention Relate to Behavior and Attachment?" Developmental Neuropsychology, Vol. 10, 1994, pp. 179188.

[30] M. A. Stein, E. Szumowski, T. A. Blondis, et al., "Adaptive Skills Dysfunction in ADD and ADHD Children," Journal of Child Psychology and Psychiatry, Vol. 36, No. 4, 1995, pp. 663-670.

[31] T. P. Zahn, M. Kruess and J. L. Rapoport, "Reaction Time Indices of Attention Deficits in Boys with Disruptive Behavior Disorders," Journal of Abnormal Child Psychology, Vol. 19, No. 2, 1991, pp. 233-252.

[32] M. J. Owen, C. D. Baldwin and V. M. Howie, "Incidence and Duration of Otitis Media with Effusion during the First Two Years of Life," Paper Presented at: Southern Society for Pediatric Research, New Orleans, 4-5 February 1988.

[33] R. M. Lerner, M. Palermo, A. Spiro, et al., "Assessing the Dimensions of Temperamental Individuality across the Life Span: The Dimensions of Temperament Survey (DOTS)," Child Development, Vol. 53, No. 1, 1982, pp. 149-159.

[34] R. L. Thorndike, E. P. Hagen and J. M. Sattler, "StanfordBinet Intelligence Scale," 4th Edition, Riverside Publishing, Chicago, 1986.

[35] T. M. Achenbach, "Manual for the Child Behavior Checklist/2-3 and 1992 Profile," University of Vermont Department of Psychiatry, Burlington, 1992.

[36] T. M. Achenbach, "Manual for the Child Behavior Checklist/4-18 and 1991 Profile," University of Vermont Department of Psychiatry, Burlington, 1991. 
[37] T. M. Achenbach, "Manual for the Teacher's Report Form and 1991 Profile," University of Vermont Department of Psychiatry, Burlington, 1991.

[38] T. R. Dupuy, D. McCarney and L. M. Greenberg, “T.O.V.A. Manual: Tests of Variables of Attention Computer Program. Version 6.0 for the IBM PC or IBM Compatible," Lawrence M. Greenberg, Minneapolis, 1990.

[39] A. B. Hollingshead, "Hollingshead Four-Factor Index of Social Status," Department of Sociology, Yale University, New Haven, 1975.

[40] B. M. Caldwell and R. H. Bradley, "Home Observation for Measurement of the Environment (Revised Edition)," University of Arkansas, Little Rock, 1984.

[41] K. G. Joreskog and D. Sorbom, "LISREL 7: Estimation of Linear Structural Equation Systems by Maximum Likelihood Methods," National Educational Resources, Chicago, 1988.

[42] D. Francis, "An Introduction to Structural Equation Models," Journal of Clinical and Experimental Neuropsychol- ogy, Vol. 10, No. 5, 1988, pp. 623-639.

[43] T. Hubbard, J. L. Paradise, B. J. McWilliams, et al., "Consequences of Unremitting Middle Ear Disease in Early Life: Otologic, Audiologic and Developmental Findings of Children with Cleft Palate," New England Journal of Medicine, Vol. 312, No. 24, 1985, pp. 1529-1534.

[44] A. R. Adesman, L. A. Altshuler, P. H. Lipkin, et al., "Otitis Media in Children with Learning Disabilities and in Children with Attention Deficit Disorder with Hyperactivity," Pediatrics, Vol. 85, No. 3, 1990, pp. 442-446.

[45] K. Hoffman-Lawless, R. W. Keith and R. T. Cotton, “Auditory Processing Abilities in Children with Previous Middle Ear Effusion," Annals of Otology, Rhinology and Laryngology, Vol. 90, No. 5, 1981, pp. 543-545.

[46] P. A. Silva, D. Chalmers and I. Stewart, "Some Audiological, Psychological, Educational and Behavioral Characteristics of Children with Bilateral Otitis Media with Effusion: A Longitudinal Study," Journal of Learning Disabilities, Vol. 19, No. 3, 1986, pp. 165-169. 